\title{
Conocimiento educativo en el contexto escolar y en la educación familiar mapuche: principales tensiones epistemológicas*
}

\author{
SEGUNDO ENRIQUE QUINTRIQUEO MILLÁN \\ Universidad Católica de Temuco, Temuco, Chile \\ ENRIQUE HERNÁN RIQUELME MELLA \\ Universidad Católica de Temuco, Temuco, Chile \\ SOLEDAD MORALES SAAVEDRA \\ Universidad Católica de Temuco, Temuco, Chile \\ DANIEL QUILAQUEO RAPIMÁN \\ Universidad Católica de Temuco, Temuco, Chile \\ MARITZA CECILIA GUTIÉRREZ SURJAN \\ Universidad Católica de Temuco, Temuco, Chile
}

\section{RESUMEN}

En este artículo se comparan perspectivas sobre la integración del conocimiento mapuche, en la educación escolar y en la educación familiar, desde la perspectiva de padres, estudiantes y profesores de escuelas de la Novena Región de La Araucanía, Chile. Aborda las implicancias de un currículum y educación monocultural sobre la formación de niños mapuches, que presentan lógicas diferentes de saberes y conocimientos educativos. La metodología empleada es la investigación educativa, con un diseño descriptivo-comparativo. Participaron 455 estudiantes, 39 profesores, 148 padres y 48 sabios de las áreas Lafkenche y Pewenche. Los resultados muestran diferencias en la apreciación del conocimiento educativo mapuche en la escuela y familia, según territorialidad, roles y dominio de la lengua mapuche. Se concluye que las prácticas pedagógicas contextualizadas a las territorialidades constituyen una alternativa para generar el diálogo de saberes, desde el enfoque educativo intercultural en la formación escolar.

\section{PALAVRAS CLAVE}

conocimiento indígena; conocimiento escolar; currículum escolar; epistemología contextual; educación intercultural.

* Este artículo presenta los resultados del proyecto de investigación financiado por el Fondo Nacional de Desarrollo Científico y Tecnológico FONDECYT N ${ }^{\circ} 1110489$ y el proyecto $\mathrm{N}^{\circ} 1140490$ "Conocimientos geográficos y territoriales mapuches: una base para la formulación de contenidos educativos interculturales pertinentes y contextualizados". 


\title{
EDUCATIONAL KNOWLEDGE IN THE SCHOOL CONTEXT AND IN THE FAMILY MAPUCHE EDUCATION: MAIN EPISTEMOLOGICAL TENSIONS
}

\begin{abstract}
This article describes and compares perspectives on the integration of Mapuche knowledge both in school and family education from the point of view of parents, students and education professionals at schools of the Ninth Region of La Araucanía, Chile. The implications of a monocultural curriculum and training of Mapuche children were analysed, considering different logics of knowledge and educational abilities. The methodology used was a descriptive-comparative educational research. The study involved 455 students, 39 education professionals, 148 parents, and 48 sages from Lafkenche and Pewenche territorial areas. Results show differences in valuing Mapuche educational knowledge in schools and inside families, including territory concept, roles, and Mapuche language domain. We conclude that contextualized pedagogical practices may be an alternative to create a dialogue of knowledge on the basis of intercultural educational approach in school formation.
\end{abstract}

\section{KEYWORDS}

indigenous knowledge; school knowledge; school curriculum; contextual epistemology; intercultural education.

\section{CONHECIMENTO EDUCATIVO NO CONTEXTO ESCOLAR E NA EDUCAÇÃO DA FAMILIA MAPUCHE: PRINCIPAIS TENSÕES EPISTEMOLÓGICAS}

\section{RESUMO}

$\mathrm{O}$ artigo descreve e compara perspectivas sobre a integração do conhecimento Mapuche, tanto na educação escolar e familiar, a partir da perspectiva dos pais, alunos e professores de escolas da Nona Região de La Araucanía, Chile. São analisadas as implicações de um currículo de educação monocultural e treinando crianças mapuche, tendo diferentes lógicas de conhecimentos e habilidades educacionais. A metodologia utilizada é a pesquisa educacional, com um projeto descritivo-comparativa. Envolvido 455 alunos, 39 professores, 148 pais e 48 sábios das áreas territoriais Lafkenche e Pewenche. Os resultados mostram diferenças na valorização do conhecimento educacional Mapuche na escola e família, como a territorialidade, papéis e domínio da língua Mapuche. Concluímos que contextualizadas práticas pedagógicas territorialidades e o conhecimento educacional Mapuche pode constituir uma alternativa para gerar um diálogo de saberes, a partir da abordagem educativa intercultural na formação escolar.

\section{PALAVRAS-CHAVE}

conhecimento indígena; o conhecimento escolar; currículo escolar; a epistemologia contextual; educação intercultural. 


\section{INTRODUCCIÓN}

La investigación sostiene que la escolarización en contexto indígena es un proceso que abarca toda la vida, realizada en base a contenidos y finalidades educativas fundadas en un currículum escolar monocultural, centralizado y homogeneizante. Es un currículum diseñado para satisfacer las necesidades de aprendizaje que se generan en el ámbito cultural, social y económico de la sociedad nacional. Por otra parte, sostenemos que en contexto de comunidades mapuches aún existe una educación familiar, que permite la transmisión de un conjunto de contenidos que dotan a las nuevas generaciones de una lógica del saber y del conocimiento, sea en forma consciente o inconsciente, pero que les permite comprender la realidad sociocultural (Quintriqueo y Torres, 2012).

En esa perspectiva sostenemos que, los kimches (sabios) de familias y comunidades mapuches de las distintas territorialidades, han adaptado sistemas de saberes, contenidos y finalidades educativas, vigentes y válidas, para la formación de las personas en relación con la sociedad occidental. Esta relación podría variar, según las áreas territoriales, por efecto de las relaciones interétnicas y la diversidad de elementos que definen las unidades y procesos territoriales, generando variaciones culturales asociadas al dominio del conocimiento educativo propio, para la formación de personas (Quintriqueo, 2011-2013). Sin embargo, se observa una coexistencia del conocimiento mapuche y el conocimiento escolar en ambas territorialidades, que son desconocidos u omitidos en las prácticas educativas escolares. Este hecho podría obstaculizar el aprendizaje significativo de los estudiantes en el contexto escolar y la capacidad para desarrollar habilidades de interacciones y relaciones sociales, a causa del racismo, los estereotipos y los prejuicios históricos que operan como desprecio epistemológico al valor educativo del conocimiento mapuche en el contexto escolar.

En ese marco, el explicitar la presencia de saberes mapuches y su distancia con el conocimiento escolar, permitirá sustentar una base epistemológica que fundamente una relación de saberes desde la educación intercultural y el desarrollo de un currículum cada vez más intercultural y contextualizado a las áreas territoriales de la región de La Araucanía (Quintriqueo, 2014-2017). De esta forma, el objetivo de este trabajo es describir y comparar perspectivas en dos dimensiones centrales: el conocimiento mapuche en el medio escolar de escuelas situadas en comunidades, así como la mantención del conocimiento mapuche en la familia, a través de un proceso de relación de saberes, considerando el punto de vista de padres, estudiantes, profesores y miembros de la familia y la comunidad mapuche actual.

\section{MARCO DE REFERENCIA}

En la sociedad moderna, la escolarización se inscribe en el marco de un currículum escolar, donde se establece el recorrido de formación que deben vivir los estudiantes, en todos los niveles. En este sentido, cuando hablamos de escolaridad, se hace referencia a la educación recibida en la escuela, en diferentes niveles y 
complejidades, formalizada por un programa de estudios y reconocida en el marco de un establecimiento escolar legitimado y reglamentado, según la evolución de la sociedad y los sistemas educativos (Briand y Chapoulie, 1993). En este proceso, tanto estudiantes como profesores aprenden en una relación con el saber que está establecido en el marco del currículum escolar, donde los objetos de saber están definidos e institucionalizados en la escuela. Así, desde un análisis global, los principales fines de la escuela son:

1. Formalizar mecanismos de aprendizaje y asegurar la posibilidad de su realización;

2. Generar aprendizajes durables y sólidos, en función de objetivos y en una relación estructural con los medios utilizados;

3. Institucionalizar el conocimiento escolar en la sociedad a través de la formación de niños como una responsabilidad exterior a la familia; y

4. Legitimar social, ética, política y legalmente a la escuela como responsable de la instrucción en el marco de la organización escolar.

En contexto mapuche, estos fines se realizan en base a principios que orientan la escuela como institución, desde la matriz cultural fundada en la monoculturalidad euro-céntrica occidental como paradigma sociocultural dominante. En este sentido, la escuela y la educación, se han desarrollado en base a sus hitos fundadores: colonizar y producir la inferiorización de los indígenas en América Latina y particularmente en la región de La Araucanía (Quintriqueo et al., 2014).

Entonces, desde la instalación de la escuela y la educación escolar en la región de La Araucanía, con el patrocinio de las primeras escuelas misionales desde 1883, los niños han iniciado un proceso de desarraigo de la educación familiar (Quintriqueo, 2010). En este proceso, los internados y las escuelas en tanto instituciones sociales, son percibidas por la población mapuche como 'una oportunidad de movilidad social y económica' en tanto favorecería la formación de un capital humano preparado para el desarrollo socioeconómico, político, social y educacional de las familias y comunidades (UNICEF, 2006). Sin embargo, desde sus inicios la escuela y la educación en contexto mapuche, es concebida para la sociedad criolla chilena y de quienes son responsables de la configuración del Estado Nación, como un instrumento de integración social básica. También, para la dominación y 'eliminación del sujeto' mapuche del escenario de desarrollo social, cultural, político y educacional del país. La concepción descrita, limita el reconocimiento y autonomía de las familias y las comunidades en el contexto regional y nacional (Quintriqueo, Quilaqueo y Riquelme, 2014).

Desde la etnografía, sostenemos que la educación escolar de los niños mapuches es desarrollada tanto en contexto rural como urbano, en un sistema educativo único nacional. En contexto rural, las escuelas en general son de nivel primario y los niños pueden cursar de $1^{\circ}$ a $8^{\circ}$ año de Educación Básica. Estas son escuelas que en su mayoría están organizadas por cursos, por aula y/o curso multigrado, donde las clases son realizadas por un solo profesor. Aquí, la matrícula es variable, entre 12 a 20 estudiantes por curso. En el contexto urbano, al igual que en el rural, la mayoría de las escuelas imparten educación de $1^{\circ}$ a $8^{\circ}$ año. Sin embargo, el número 
de estudiantes por curso puede ascender a 40, el número de profesores por aula varía, sin curso multigrado. Los niños mapuches para continuar estudios de Educación Media (secundaria), deben emigrar a escuelas situadas en contexto urbano. Tanto en contexto rural como urbano, la educación entregada a niños mapuches se basa en el currículum nacional único, organizado por asignaturas, según disciplina, como contenidos mínimos de enseñanza-aprendizaje para todos los estudiantes, mapuches y no mapuches. Cabe remarcar que entre los docentes que imparten las clases son de origen mapuche y no mapuche. Sin embargo, los profesores de origen mapuche al ser formados por el sistema educativo único nacional monocultural no poseen la formación para incluir los contenidos de la educación familiar mapuche en sus prácticas educativas.

A principios del siglo XIX, los niños en general ingresaban a la escuela con un fuerte arraigo a su lengua vernácula, a sus costumbres, tradiciones, sólidos valores educativos mapuches fundados en el conocimiento sobre sus parientes y la historia de su comunidad (Guevara, 1913). Sin embargo, en la actualidad, la situación sociocultural ha cambiado, debido al proceso histórico de integración de la familia y la comunidad a la sociedad nacional. En este proceso, mediante la escolarización, los niños debieron someterse a la evangelización, al aprendizaje de la lengua castellana, las costumbres, valores, saberes y conocimientos transmitidos por la escuela como cultura escolar (Rockwell, 1995). Como resultado de la escolarización basada en un currículum monocultural y monolingüe en castellano, las nuevas generaciones desde mediados del siglo XX, abandonan progresivamente sus costumbres, creencias, saberes, conocimientos y valores sociales y culturales vitales, por ejemplo la lengua mapunzugun (Quintriqueo et al., 2015).

Las escuelas instaladas en comunidades mapuches, desde principios del siglo XIX (Poblete, 2001), se orientaron principalmente a la enseñanza del castellano, la adquisición de destrezas para la lectura, la escritura y operaciones aritméticas básicas. Desde entonces ha sido valorada por las familias y las comunidades mapuches, como estrategia de alfabetización, para evitar en las nuevas generaciones los abusos cometidos en su contra. Además, históricamente ha constituido un espacio de relaciones interétnicas entre la población mapuche y no mapuche. En este sentido, desde mediados del siglo XIX (1920), las demandas y exigencias históricas se identifican con claridad, donde los mapuches buscan ser responsables de su educación, en escuelas donde pueda enseñarse el idioma propio, el mapunzugun (Alvarez-Santullano y Forno, 2008).

En las últimas décadas, como respuesta a la educación monocultural, se observa el desafío de un proceso de reidentificación mapuche, donde los niños son inducidos por sus padres y dirigentes de organizaciones mapuches a ser formados en la educación familiar, desde saberes y conocimientos educativos asociados a la sabiduría ancestral. La sabiduría ancestral refiere a conocimientos de prácticas de la medicina tradicional, relación de respeto con la naturaleza, las prácticas socio religiosas, métodos educativos que se preservan en la memoria social, conocimientos sobre el territorio, la geografía y la lengua propia. Estos saberes, actualmente son revalorizados y puestos en posición de igualdad para la formación de las nuevas generaciones, tanto en el medio familiar como en el medio escolar. 
Actualmente se observa que, desde 1994, existe una iniciativa gubernamental por impulsar la creación del Programa de Educación Intercultural Bilingüe (PEIB), fundamentado en los planteamientos de la Ley Indígena 19.253 de 1993. Además, existen instrumentos de derecho internacional como la Declaración de Naciones Unidas sobre los Derechos de los Pueblos Indígenas (UNESCO, 2007) y la Declaración de los Derechos del Niño (UNICEF,2006), que demandan una educación más contextualizada a la realidad sociocultural y lingüística de los estudiantes de ascendencia indígena. En estas normativas se reafirma que en el ejercicio de sus derechos, los pueblos indígenas deben estar libres de toda forma de discriminación y se reconoce además, que las familias y comunidades indígenas tienen que compartir la responsabilidad por la crianza, la formación, la educación y el bienestar de sus hijos, en consonancia con sus derechos. En ese marco, según la política pública en educación, Chile es un país multicultural y plurilingüe, en el cual convergen una diversidad de culturas y sistemas lingüísticos. Este hecho impone el desafío de convertir la escuela en un espacio educativo, en el cual se asegure a niños y niñas de culturas y lenguas diferentes, el acceso a oportunidades de aprendizaje de las lenguas indígenas, de modo sistemático y pertinentes a su realidad, de acuerdo con sus costumbres, valores y cosmovisión propia (Ministerio de Educación [MINEDUC],2009).Lo anterior contribuiría a lograr relaciones más equitativas entre las personas y los grupos sociales en nuestro país.

Sin embargo, la educación escolar en contexto mapuche continúa centrada en la epistemología de la monoculturalidad. Este problema se traduce en una distancia entre los conocimientos vernáculos y aquellos conocimientos transmitidos e institucionalizados en la escuela para entregar una formación escolar. Es lo que planteamos como distancia epistemológica entre el conocimiento mapuche y el conocimiento escolar en estudiantes de las áreas territoriales mapuches, lo que se refleja en problemas centrales vinculados con los tipos de saberes y los criterios de verdad. Sostenemos que el repertorio de experiencias educativas y socioculturales son las fuentes de orígenes del saber y conocimiento, principalmente en contextos indígenas, donde el conocimiento es construido en una relación directa del sujeto con el saber, con el conocer y el objeto de saber (Falavigina y Arcanio, 2011). Así, en la educación escolar en contexto mapuche, los criterios y razones epistemológicas que orientan el desarrollo de la educación, se sustentan en el conocimiento disciplinario de los distintos sectores de aprendizaje del currículum escolar (Quintriqueo y Maheux, 2004). En ese sentido, en el currículum quedan ocultadas o negadas las experiencias educativas y socioculturales acumuladas en la memoria individual y social de las familias, a través del tiempo y expresada en los discursos orales o escritos, según las áreas territoriales mapuches (Quintriqueo y Muñoz, 2012). De esta forma, la vivencia de la territorialidad desde la cosmovisión mapuche, trae consigo un conocimiento que por ejemplo, desafía las ideas occidentales de tiempo y espacio, el conocimiento sobre el mundo natural y espiritual como el conocimiento sobre la historia local (Quilaqueo, Quintriqueo y Cárdenas, 2005; Quintriqueo y Cárdenas, 2010), los cuales son ignorados en las tres dimensiones del currículum escolar: prescrito, real y oculto.

Aquí entendemos el currículum prescrito como el conjunto de orientaciones, definición de categorías de contenidos y finalidades educativas, progresión de competencias conceptuales, actitudinales y procedimentales definidas por las bases 
curriculares del sistema educativo, establecidos en sus respectivos niveles de educación Preescolar, Básica y Media. El currículum real corresponde a aquellos saberes, actitudes, ideologías, creencias, intereses y finalidades educativas que el profesor transmite en el desarrollo de sus prácticas educativas escolares, sea planificada o no, consciente o inconscientemente. $Y$, el currículum oculto se relaciona con todos los procesos de negociación entre el profesor y los estudiantes, entre los propios estudiantes y éstos a su vez con los miembros de su familia y comunidad, donde se transmiten, sin siquiera proponérselo, saberes, actitudes, ideologías, creencias, intereses sobre la educación, la cultura y la sociedad (Perrenoud, 1994; Quintriqueo y Mahuex, 2004; UNESCO, 2005, 2007).

En ese marco curricular, promover en los estudiantes los conocimientos y discursos del mundo académico, sin establecer la diferenciación contextual constituye un problema para la educación, especialmente con la racionalidad monocultural establecida en las dimensiones del currículum escolar (Izquierdo, 1999; García, 2007). Este es un problema complejo de superar, principalmente a nivel epistemológico, ya que es necesario visualizar las incumbencias que aparecen entre las reglas y normas de validación de cada grupo social en la construcción y realización del currículum en la escuela. Visualizar escuelas donde confluyen diferentes epistemologías o pluralismo epistemológico (Olivé, 2009), donde se supere la descalificación de los saberes mapuches, para iniciar un nuevo recorrido dialógico, crítico y basado en la relación de saberes, en el que los puntos de divergencia entre opciones epistemológicas sean posibles, es fundamental para avanzar en el entendimiento de nuestras diversidades de saberes y conocimientos (Mato, 2008).

En este sentido, la legitimación de los saberes y conocimientos mapuches en la escuela, no solo debe darse en instancias netamente metodológicas. Esto porque el significado y sentido del saber, lejos de fijarse en lo puramente objetivo o subjetivo, se configura a través de procesos en espirales recursivos del dialógico cultural y como prácticas socioculturales epistémicas que deberán ser integradas en el marco de la institución escolar (Quintriqueo, Quilaqueo y Riquelme, 2014). En esta perspectiva, los aspectos centrales de una epistemología contextual e intercultural se fundamentan en los siguientes supuestos:

1. La realidad es una construcción social que no existe independiente de los actores, del contexto y las teorías implícitas que le dan forma, donde la lengua permite conceptualizarla y comunicarla desde una lógica propia;

2. Toda objetividad es objetividad a partir de una versión, más o menos eficaz para comprender la realidad;

3. Las interpretaciones son subjetivas en base a una serie de enunciados, cuyo sentido y estatus referencial están asociados a explicaciones de la realidad sociocultural y las posiciones de los sujetos en los actos individuales y sociales, en un contexto específico;

4. Los valores y las verdades responden a orígenes y realidades diversas no tienen un origen unidimensional y exclusivamente euro-céntrico, sino que, están asociados a la historia individual y social de los sujetos en cada uno de sus contextos de vida, lo cual obliga a confrontar todo juicio de valor de carácter hegemónico universalista; $y$ 
5. El conocimiento es un hecho político, puesto que las categorías y los valores sociales son el resultado de actividades sociales, construido en relaciones directas y concretas con el medio natural, social, cultural y espiritual (Morin, 1998; Mato, 2008; Quintriqueo y Muñoz, 2012).

Esto implica comprender que, desde la epistemología contextual e intercultural, la cultura mapuche tiene formas particulares de entender y significar el mundo que son igualmente válidas, en tanto constituyen el conjunto de conocimientos, valores y creencias que dan sentido a la existencia de un pueblo y por tanto, a la de las personas que lo integran (Schmelkes, 2008).

\section{METODOLOGÍA}

La metodología utilizada es la investigación educativa, con el propósito de construir conocimientos como una base para comprender y mejorar la educación en contextos interculturales, fundamentados en el enfoque multimétodo (Gauthier, 1998; Gauthier, Martineau y Desbiens, 1997; Rodríguez, Gil y García, 1999; Bisquerra, 2004; Ruiz,2008). En este proceso se utilizan dos o más procedimientos para la indagación sobre un mismo fenómeno u objeto de estudio a través de los diferentes momentos del proceso de investigación. Su utilización se justifica, ya que el interés de la presente investigación se asocia a la complejidad que presenta la relación entre el conocimiento mapuche y el conocimiento escolar.

\section{PARTICIPANTES}

La investigación se desarrolló en la región de La Araucanía, ubicada en la zona Centro-Sur de Chile, en el contexto de escuelas situadas en comunidades mapuches rurales y semi-rurales de las territorialidades Perwenche - sector cordillerano - y Lafkenche - sector costero. Todos los participantes accedieron voluntariamente a ser parte de la investigación.

La muestra consideró un total de 14 escuelas ubicadas en La Araucanía. Desde estas escuelas se seleccionaron de manera no probabilística 455 estudiantes, 39 profesores y 148 padres, sumando una muestra total de 642 personas. Del total de personas, 169 fueron "Lafkenches" y 473 "Perwenches". Una característica importante de los participantes es el conocimiento del mapunzugun; $14,5 \%$ habla mapunzugun, $34,3 \%$ entiende la lengua y 50,9\% no habla la lengua. Además de los participantes señalados, se incluyó a 48 Kimches de ambos territorios, 24 en cada territorialidad, respondieron una entrevista semi-estructurada.

\section{INSTRUMENTOS}

Cuestionario sobre conocimiento mapuche en la escuela y educación familiar: compuesto por 12 ítems, que incluyen diferentes proposiciones en las que los participantes pueden mostrarse en desacuerdo, o de acuerdo. Este cuestionario fue desarrollado desde entrevistas previas a distintos actores sociales de la comunidad: 
profesores, estudiantes y padres para explorar la percepción de estos participantes en relación a la integración del conocimiento mapuche en la escuela y en la familia. De esta forma, se organizaron teóricamente dos dimensiones: integración del conocimiento mapuche en la escuela e integración del conocimiento mapuche en la educación familiar.

- Integración del conocimiento mapuche en la escuela: explora la integración del conocimiento y significado de las actividades culturales mapuches en la escuela, por ejemplo, narraciones sobre la historia mapuche, mapunzugun y conocimiento mapuche en la escuela. Entre los ítems de esta dimensión se encuentran "En la escuela se enseñan conocimientos mapuches como la historia de la comunidad, el lakutun y la artesanía tradicional" y "En la escuela invitan a ancianos o kimches - sabios -, para que nos enseñen la historia de la comunidad o de las familias mapuches". Esta dimensión incluyó 6 ítems, pudiendo obtener un puntaje máximo de 6 Pts. De esta forma, a mayor puntaje en esta dimensión, mayor acuerdo con la integración del conocimiento social y cultural mapuche en la escuela. El índice de confiabilidad para esta dimensión fue de 0.74 .

- Integración del conocimiento mapuche en la educación familiar: la educación familiar es entendida como un modelo de formación encargado de transmitir saberes y conocimientos que contribuyan a fortalecer el patrimonio cultural. Desde esta base, la dimensión explora la percepción sobre la integración y transmisión de conocimientos mapuches en la educación familiar. Entre los ítems que componen la dimensión encontramos: "Mis padres o abuelos me enseñan sobre la historia de la familia, por ejemplo, si ha habido logko, machi" y "Mis padres o abuelos me cuentan sobre los viajes que antiguamente realizaban los mapuches, por ejemplo a comunidades cercanas o a la Argentina”. Esta dimensión incluyó 6 ítems, pudiendo obtener un puntaje máximo de 18 Pts. De esta forma, a mayor puntaje en esta dimensión, mayor acuerdo con la integración y mantención del conocimiento mapuche en la familia. El índice de confiabilidad para esta dimensión fue de 0.73 .

El instrumento fue desarrollado para ser respondido por estudiantes, profesores y padres, de modo que la presentación de los ítems se organizó desde la perspectiva de cada grupo, por ejemplo, para los estudiantes el ítem "los profesores usan mi conocimiento mapuche sobre naturaleza" correspondió para los padres al ítem "los profesores usan el conocimiento mapuche que mi hijo tiene sobre la naturaleza"; y para los profesores "como profesor uso el conocimiento mapuche que mis estudiantes tienen sobre la naturaleza".

El cuestionario consideró además, la identificación étnica de los participantes, como mapuche o no mapuche, así como al dominio del mapunzugun (habla, entiende y no habla) y, finalmente, la territorialidad, es decir, el sector geográfico con el que se identifica y reside (Lafkenche o Perwenche).

Entrevista semi-estructurada: En relación a los mismos temas abordados en la escala, se aplicó una entrevista semiestructurada a 48 kimches, con preguntas 
abiertas, para identificar categorías de saberes y conocimientos educativos explícitos e implícitos. La entrevista incluyó preguntas similares a las que se señalaron en la estructura del cuestionario, pero las características de esta estrategia permitían explorar con más detalle el contenido abordado. El propósito de usar esta entrevista fue el complementar la interpretación cuantitativa de los datos del cuestionario aplicado a estudiantes, profesores y padres, esta vez desde el punto de vista de los kimches de la comunidad, quienes son considerados sabios portadores de los conocimientos y saberes mapuches (Quilaqueo y Quintriqueo, 2010).

\section{TÉCNICAS Y PROCEDIMIENTOS DE ANÁLISIS}

En consideración al objetivo planteado, se implementó un diseño descriptivo-comparativo. El análisis cualitativo de la información se realizó mediante el análisis de contenido, que busca relevar núcleos de saberes centrales de orden abstracto, dotados de sentido y significado desde la perspectiva de los productores del discurso (Krippendorff, 1990). Este proceso incluye una codificación triangulada de contenidos, para una posterior ponderación de su relevancia diferenciada de acuerdo a las distintas categorías de análisis y conglomerados, desde la codificación abierta y axial, para avanzar en la construcción de una teoría fundamentada sobre la distancia entre el conocimiento cultural mapuche y el escolar (McMillan y Schumacher, 2005). El análisis cuantitativo de los datos se realizó a través de estadística descriptiva y de comparación de grupos. La naturaleza de las variables, y los análisis de normalidad realizados permiten análisis de comparación no-paramétricos, de modo que las comparaciones se realizaron usando pruebas U de Mann-Whitney y $\mathrm{H}$ de Kruskal-Wallis.

\section{RESULTADOS}

Los resultados se presentan en tres categorías:

1. comparando las dimensiones del conocimiento mapuche en la escuela y educación familiar, según el territorio al que pertenecen los participantes,

2. comparando las dimensiones del conocimiento mapuche en la escuela y familia, desde el punto de vista de los estudiantes, padres de familias y profesores, $y$

3. comparando las dimensiones del conocimiento mapuche en la escuela y familia, desde el dominio de la lengua mapuche.

\section{A. DESDE LA TERRITORIALIDAD}

Al comparar las dimensiones del conocimiento mapuche en la escuela y familia, según la territorialidad, es posible observar diferencias estadísticamente significativas entre ambos sectores. La Tabla 1 presenta los rangos promedios de ambas dimensiones en relación a la territorialidad, así como los estadísticos de contraste.

Con respecto a la Integración del conocimiento mapuche en la escuela, se puede observar en la Tabla 1 que los rangos promedios señalan que las personas que asisten al colegio en el territorio Lafkenche, identifican con mayor frecuencia los intentos 
de la escuela y los profesores por integrar el conocimiento mapuche en las aulas. Lo anterior puede también estar asociado a una mirada más crítica de parte de las personas del territorio Perwenche, sobre el rol de las escuelas para la revitalización del conocimiento mapuche o efectivamente, existe un mayor esfuerzo de los profesores del territorio Lafkenche por integrar los conocimientos culturales a la escuela.

Desde el análisis cualitativo es posible apreciar que en la territorialidad Lafkenche, existen prácticas socioculturales que están a la base de los procesos de construcción del saber y conocimiento propio que permiten también hacer la distinción con el conocimiento escolar. El conocimiento sobre el territorio es un claro ejemplo, con una frecuencia del $30,6 \%$ de un total de 766 recurrencias en el discurso. De la misma forma, el código relación con el medio natural con un 19,4\% de las frecuencias en el discurso de los kimches. Esto devela la importancia de la relación hombre-naturaleza en la construcción del conocimiento, lo que indica la interdependencia del hombre con su medio. Este hecho señala su importancia para la construcción y reconstrucción del saber propio en la actualidad, lo que está influenciado por el contacto con la sociedad occidental y la coexistencia con elementos propios de ella.

En relación a la Integración del conocimiento mapuche en la educación familiar, es posible observar que las personas del territorio Lafkenche, tienden a reconocer una mayor presencia de la educación familiar en las comunidades a las que pertenecen.

En la territorialidad Perwenche, se constata el código conocimiento familiar con una frecuencia de uso del $45,5 \%$ en el discurso de kimches y padres, de un total de 1.176 recurrencias. Esto indica que en la actualidad aún existen saberes y conocimientos que son construidos en el medio familiar, los cuales son ocultados tanto en el contexto escolar como social. En segundo lugar se ubica el código pérdida del conocimiento mapuche con un 34\% de la recurrencia en el discurso, lo que indica que se han perdido algunos saberes y conocimientos, principalmente en las nuevas generaciones. Finalmente, el código conocimiento escolar obtiene un $20,5 \%$ de las recurrencias, lo que indicaría, que la escuela y el conocimiento que allí se enseña están institucionalizados en las personas, en las familias y en las comunidades mapuches, para desarrollarse en la sociedad actual. Este hecho, también estaría influyendo en la pérdida de saberes y conocimientos mapuches, dado el carácter monocultural de la enseñanza que se imparte hoy día en las escuelas del territorio Perwenche.

En tanto, en la territorialidad Pewenche se constata que el código Educación Familiar obtiene la más alta frecuencia, con un $63 \%$ de uso en el discurso de los

Tabla 1 - Rangos medios del conocimiento mapuche en la escuela y la educación familiar

\begin{tabular}{l|c|c|c|c|c}
\hline \multirow{2}{*}{ Dimensión } & \multicolumn{2}{|c|}{ Rango Medio } & \multirow{2}{*}{ U } & \multirow{2}{*}{ Z } & \multirow{2}{*}{$P$} \\
\cline { 2 - 3 } & Lafkenche & Pewenche & & & \\
\hline Conocimiento mapuche en la escuela & 392,70 & 295,53 & 27686,000 & $-5,907$ &, 000 \\
\hline Educación Familia mapuche & 360,95 & 307,40 & 33301,000 & $-3,267$ &, 001 \\
\hline
\end{tabular}


kimches, de un total de 1.176 recurrencias, lo que indica la existencia de saberes y conocimientos enseñados en la familia mapuche. Asimismo, se identifican otros dos códigos:

1. Contenidos educativos, el cual alcanza la más alta frecuencia con un $67,6 \%$, asociados a conocimientos sobre el medio social y natural; y

2. Prácticas socioculturales, alcanza un $32,4 \%$ de las frecuencias, asociadas al conocimiento con respecto a las tradiciones y costumbres mapuches del sector Perwenche.

\section{B. DESDE LOS ROLES DE LOS PARTICIPANTES}

Al comparar la aproximación que los participantes, en sus roles como estudiantes, padres o profesores, tienen en relación al conocimiento escolar y educación familiar, es posible observar diferencias estadísticamente significativas. La Tabla 2 presenta los rangos promedios de ambas dimensiones según la perspectiva de cada participante, así como la correspondiente prueba de comparación y la significación.

Con respecto a la Integración del conocimiento mapuche en la escuela, se puede observar en la Tabla 2 que un análisis por pares en la primera dimensión, es posible observar diferencias estadísticamente significativas entre los estudiantes (Rango promedio $=242,70$ ) y los profesores (Rango promedio=297,08). $\mathrm{U}=6900,00 . p<, 05$.

Los profesores tienen una visión más positiva de su rol en la integración del conocimiento mapuche en el ámbito escolar (coherente con lo observado por Quintriqueo, Quilaqueo y Riquelme, 2014), que discrepa con la visión que tienen los estudiantes. Por ejemplo, ante la pregunta "Cuando el profesor enseña las materias, incorpora costumbres mapuches para ejemplificar el conocimiento escolar", el 87\% de los profesores responde afirmativamente, a diferencia del 51,4\% de los estudiantes. Lo mismo sucede con la afirmación "En la escuela enseñan conocimientos mapuches como la historia de la comunidad, el lakutun (padrinazgo) y la artesanía tradicional", donde el $76,9 \%$ de los profesores que responde afirmativamente a diferencia del $44 \%$ de estudiantes.

En relación a la Integración del conocimiento mapuche en la educación familiar, en un análisis por pares en la segunda dimensión, es posible observar diferencias estadísticamente significativas entre los estudiantes (Rango promedio $=290,32$ ) y los padres (Rango promedio=337,91). $\mathrm{U}=28355,00 . p<, 01$.

En esta dimensión es posible observar un descenso paulatino y marcado de aproximación al conocimiento cultural mapuche, desde los padres a los profesores.

Tabla 2 - Rangos medios del conocimiento mapuche, según el rol de los participantes

\begin{tabular}{l|c|c|c|c|c|c}
\hline \multirow{2}{*}{ Dimensión } & \multicolumn{3}{|c|}{ Rango Medio } & \multirow{2}{*}{ H } & \multirow{2}{*}{ Gl } & Sig. \\
\cline { 2 - 4 } & Estudiantes & Padres & Profesores & & & \\
\hline $\begin{array}{l}\text { Conocimiento mapuche } \\
\text { en la escuela }\end{array}$ & 309,48 & 340,96 & 379,32 & 7,50 & 2 &, 023 \\
\hline Educación Familia mapuche & 308,12 & 359,30 & 334,12 & 8,94 & 2 &, 011 \\
\hline
\end{tabular}


Por ejemplo, ante la afirmación "mis padres o abuelos me cuentan sobre los viajes que antiguamente realizaban los mapuches" el $65,5 \%$ de los padres se muestra de acuerdo, el 52,3\% de los estudiantes y el 30,8\% de los profesores.

Padres y estudiantes observan una mayor distancia entre los conocimientos culturales mapuches y los que se enseñan en la escuela, a diferencia de los profesores, para quienes se hacen esfuerzos por integrar el conocimiento cultural mapuche en el aula. Al mismo tiempo, es posible observar una pérdida generacional paulatina de transmisión de conocimientos culturales, donde los padres se acuerdan -en mayor porcentaje- que se les enseñó sobre distintos ámbitos del conocimiento mapuche. Por ejemplo, la naturaleza, geografía, y actividades culturales, mientras que los estudiantes se muestran más distantes de esta afirmación y los profesores aún más. Según Quintriqueo, Quilaqueo y Riquelme (2014), esto puede significar que progresivamente en la familia se enseña en menor grado la lengua y los conocimientos mapuches. A partir de lo señalado, se establece que en la actualidad un alto porcentaje de los niños mapuches no están aprendiendo normas de convivencia en el medio familiar. O bien, podría indicar que los procesos de escolarización actual, aún ocultan o niegan la existencia de la educación familiar mapuche sustentada en conocimientos propios.

\section{DESDE EL DOMINIO DEL MAPUNZUGUN}

Uno de los elementos que consideramos transversal para la educación familiar y escolar es la lengua; es en el idioma en el que las culturas mantienen y proyectan sus tradiciones y conocimientos, en particular la cultura mapuche que es básicamente una cultura centrada en la transmisión oral del conocimiento. Partiendo de esta premisa, se realizó un análisis de las dimensiones comparando las respuestas dadas por las personas que hablan, entienden o no hablan mapunzugun (lengua mapuche). La Tabla 3 presenta los rangos promedios de ambas dimensiones según la perspectiva de cada grupo, así como la correspondiente prueba de comparación y la significación.

Con respecto a la Integración del conocimiento mapuche en la escuela, los resultados expuestos en la Tabla 3 indican que las personas que están inmersas en la lengua tienen una perspectiva distinta sobre la integración o exclusión de distintas dimensiones del conocimiento cultural mapuche en la escuela.

En esta dimensión, las personas que hablan el mapunzugun están de acuerdo en un $80,6 \%$ en que existen profundas diferencias entre el conocimiento mapuche

Tabla 3 - Rangos medios del conocimiento mapuche en la escuela y la familia, según el dominio del mapunzugun de los participantes

\begin{tabular}{|c|c|c|c|c|c|c|}
\hline \multirow{2}{*}{ Dimensión } & \multicolumn{3}{|c|}{ Rango Medio } & \multirow{2}{*}{ H } & \multirow{2}{*}{ Gl } & \multirow{2}{*}{ Sig. } \\
\hline & Habla & Entiende & No habla & & & \\
\hline $\begin{array}{l}\text { Conocimiento mapuche } \\
\text { en la escuela }\end{array}$ & 383,03 & 378,11 & 262,11 & 65,34 & 2 &, 000 \\
\hline Educación Familia mapuche & 419,79 & 384,28 & 249,35 & 104,31 & 2 & ,000 \\
\hline
\end{tabular}


y occidental, en comparación al 60,9\% de las personas que no hablan la lengua. Aun reconociendo esta distancia, es posible apreciar que aquellos que entienden y hablan la lengua mapunzugun pueden 'ver' los esfuerzos que el profesor hace para intentar construir un puente entre el conocimiento occidental y el mapuche. Por ejemplo, el 68,8\% de quienes hablan y el $66,8 \%$ de quienes entienden la lengua, pueden observar que el profesor hace esfuerzos por integrar las costumbres mapuches en relación a ejemplos sobre el conocimiento escolar, mientras que solo el 44,3\% de quienes no hablan mapunzungun logran 'ver' esta práctica. En este mismo sentido se observan diferencias en áreas específicas, tales como la enseñanza de ciencias o geografía, donde el 65,6\% de quienes hablan mapunzugun reconocen esfuerzos por integrar ejemplos de la cultura mapuche en la enseñanza, a diferencia del 41,6\% que no habla y reconoce estos esfuerzos.

En un análisis por pares en esta dimensión, es posible observar diferencias estadísticamente significativas entre quienes hablan (Rango promedio $=270,60)$ y quienes no hablan mapunzungun (Rango promedio=192,71), $\mathrm{U}=9523,500 . p<, 01$. $\mathrm{A} 1$ igual que entre quienes entienden (Rango promedio $=332,64$ ) y quienes no hablan la lengua (Rango promedio=233,59), $\mathrm{U}=22850,000$. $p<, 01$.

En la dimensión Integración del conocimiento mapuche en la educación familiar un análisis por pares de las diferencias es posible observar diferencias estadísticamente significativas entre quienes hablan (Rango promedio $=297,01)$ y quienes no hablan mapunzungun (Rango promedio=185,90), $\mathrm{U}=7160,500 \cdot p<, 01$. Al igual que entre quienes entienden (Rango promedio $=343,18$ ) y quienes no hablan mapunzungun (Rango promedio=227,45), $\mathrm{U}=20749,500$. $p<, 01$.

El conocimiento familiar también es destacado entre aquellos que están inmersos en la lengua, donde el $77,4 \%$ de quienes hablan y el 75,5\% de quienes entienden la lengua, discuten sobre la historia familiar, mientras que solo el $55 \%$ de quienes no hablan están de acuerdo con esta afirmación. Lo anterior puede estar asociado a la participación activa de los niños en actividades familiares propias de la cultura, tales como siembra, o ceremoniales, donde el 80,6\% de quienes hablan se muestran activos, y solo el $48 \%$ de quienes no hablan $m a-$ punzugun lo hace.

En resumen, los resultados señalan que el mapunzugun en la escuela está prácticamente erradicado como resultado de la escolarización occidental monocultural euro-céntrica y monolingüe en castellano e incluso, aquellos niños que saben hablarlo, señalan que no tienen los espacios para hacerlo en clases. El triángulo 'lengua, cultura y educación' se hace evidente en el análisis de esta tercera dimensión, en el que hemos podido observar que el sentido que se le da al conocimiento y la enseñanza varía entre quienes hablan y no hablan la lengua. De manera esperable, el conocimiento familiar y cultural es fundamental y profundo en los primeros, mientras que el mismo conocimiento es minimizado u ocultado entre quienes no hablan y sistemáticamente excluido de la escuela, lo mismo que los espacios en la educación para mantener vigente la lengua y la cultura. La reducción de los espacios para la interacción en la lengua mapuche en la escuela, parece ser un buen resumen de la exclusión del conocimiento cultural mapuche en la educación escolar de los niños. 


\section{DISCUSIÓN Y CONCLUSIONES}

A través de los resultados de esta investigación se constata que en escuelas situadas en contextos interculturales, la distancia epistemológica entre el conocimiento mapuche y su relación con el conocimiento escolar constituye una situación problemática que se mantiene vigente. Los resultados de esta investigación evidencian que, en la actualidad aún existen saberes y conocimientos que son construidos en el medio familiar, los cuales son ocultados tanto en el contexto escolar como social.

Las tres categorías analizadas, referidas a la distancia desde una organización del territorio, desde la valoración de los estudiantes, padres de familias y profesores, según el dominio de la lengua mapuche mapunzugun, dan cuenta que en el contexto escolar se requiere abrir la discusión de los marcos de referencia que sustentan los procesos de formación de los estudiantes mapuches y no mapuches.

$\mathrm{Al}$ respecto, entre los participantes de los contextos escolares Lafkenche y Pewenche hay diferencias sobre la evaluación de la brecha existente en la integración de conocimientos mapuches al contexto escolar. Para las personas en contexto Lafkenche, la escuela realiza mayores esfuerzos para avanzar en la superación de la distancia epistemológica, mientras que en la apreciación de las personas en contexto Perwenche, existe una evaluación crítica de las acciones de la escuela, producto de su resistencia a incorporar procesos educativos dialógicos y contextualizados. En esta dirección, también se constata que los padres y los estudiantes, indican que en las familias se desarrolla la educación familiar basada en contenidos educativos propios, aspecto que a partir de la apreciación de los profesores tiende a ser desconocido.

De acuerdo a lo anterior, es determinante que los maestros y directivos de escuelas localizadas en comunidades indígenas y rurales, conozcan las prácticas que rigen la vida comunitaria: sus creencias, tradiciones y festividades; sus formas de trabajo, de organización social y familiar; sus usos lingüísticos, y las formas de interacción entre los miembros de la comunidad y las personas ajenas a ella (Schmelkes, 2008). Lamentablemente, la reducción de los espacios para la interacción en la lengua mapuche en la escuela, parece ser un buen resumen de la exclusión del conocimiento cultural mapuche en la educación escolar de niños. Lo anterior justifica la necesidad de un enfoque de educación intercultural, contextual, crítico y dialógico, orientado a formar estudiantes conocedores de su entorno, del saber del otro, del que ha sido negado, discriminado y estereotipado, en los procesos socio-históricos a nivel local y global.

Una tensión permanente es la no consideración de las familias y de la comunidad en el trabajo educativo. Para realizar esta tarea es fundamental contar con la participación de los padres de familia y de otros miembros de la comunidad, en explicitar y sistematizar sus expectativas y necesidades específicas en relación a la educación y la escuela. En este sentido, una escuela cada vez más contextualizada, no puede ignorar a las familias y comunidades en la toma de decisiones que los afecta a todos en el medio escolar y social (Schmelkes, 2008).

Desde esa perspectiva, los resultados de la investigación permiten comprender que un aspecto clave es la disposición de los sujetos a debatir los modelos que sirven 
para construir referencias en la educación escolar. En consecuencia, se reconoce que la educación escolar necesita abrirse a una relación dialógica que permita construir finalidades y contenidos educativos que articulen los conocimientos disciplinares y los conocimientos mapuches (Quintriqueo, 2010).

Otra tensión producto de lo anterior, es la ausencia de la temática de interculturalidad en instituciones formadoras de profesores, como señala Giroux (1990), los profesores deben ser algo más que técnicos. Así, se requiere de profesionales comprometidos y capaces de superar las dificultades de la escolarización descontextualizada, a partir de una nueva relación con el saber, mediante una formación docente que considere la incorporación de contenidos culturales mapuches al currículum escolar.

Finalmente, la interculturalidad crítica pretende ser una propuesta ética y política con el objetivo de construir sociedades democráticas que articulen la igualdad y el reconocimiento de las diferentes culturas. Para ello es necesario proponer alternativas al carácter monocultural occidentalizante de la educación escolar, que predomina en la mayoría de los países latinoamericanos. Estas dos perspectivas se cruzan, se chocan y algunas veces se articulan contradictoriamente en las diversas búsquedas, experiencias y propuestas que se llevan a cabo en el continente. Esta es la principal tensión que se halla presente en el debate sobre las relaciones entre interculturalidad y educación, hoy en América Latina, y que traspasa a todas las demás (Ferrão, 2010). Esta constatación sustenta la necesidad de aprender de la biodiversidad en clave intercultural, para superar la monoculturalidad tanto en el contexto escolar como familiar y comunitario. Lo que se ratifica en el artículo 14 de la declaración de derechos de pueblos indígenas, al señalar sus derechos a establecer sistemas e instituciones que impartan educación en sus idiomas, consecuente con sus métodos culturales de enseñanza y aprendizaje.

Lo anterior es un desafío para trabajar desde epistemologías con marcos de referencias, recorridos históricos, reflexiones filosóficas y educativas distintas, desde la relación de saberes educativos mapuches y aquello establecido en el marco curricular. Esta articulación es posible, siempre que exista la disposición de transformar los modelos anclados en el esquema de la escuela tradicional, que responde a indicadores de medición cuantitativos, más que cualitativos.

Significa superar la estandarización y privilegiar más el sentido y significado de los aprendizajes escolares, aceptar que hay forma de pensar, sentir, conocer y concebir divergentes, para desenvolverse adecuadamente en la complejidad de la sociedad actual. Pensamos que los futuros ciudadanos mapuches y no mapuches, deben desenvolverse adecuadamente, como resultado de un currículum escolar intercultural para todos y no sólo para los indígenas. Para que la educación intercultural sea para todos factible, se requiere, entre otros aspectos, llevar a cabo un proceso de formación inicial y continúa de los profesores, favoreciendo el desarrollo de competencias didácticas y la adquisición de conocimientos sobre la diversidad cultural. Las acciones para la formación continua de los profesores son necesarias para construir nuevas maneras de conceptualizar la docencia, mejorar el clima escolar, así como elaborar y desarrollar proyectos educativos orientados a lograr la calidad y pertinencia de los servicios educativos. 


\section{REFERENCIAS}

Alvarez-Santullano, P.; Forno, A. La inserción de la lengua mapuche en el currículum de escuelas con educación intercultural: un problema más que metodológico. Alpha, n. 26, p. 9-28, 2008.

Bisquerra, R. Metodología de la investigación educativa. Madrid: La Muralla, 2004.

Briand, J.-P.; Chapoulie, J.-M1. L'institution scolaire et la scolarisation: une perspective d'ensemble. Revue Française de Sociologie, France, v. 34, n. 1, p. 3-42, 1993.

Falavigina, C.; Arcanio, M. Redes teóricas sobre la noción "la relación con el saber": elementos para el análisis de una noción en construcción. Revista del Instituto Rosario de Investigaciones en Ciencias de la Educación, Rosario, n. 22, p. 7-16, 2011.

Ferrão, M. Educación intercultural en América Latina: distintas concepciones y tensiones actuales. Estudios Pedagógicos, Valdivia, v. 36, n. 2, p. 333-342, 2010.

Fundo das Nações Unidas para a Infância (Unicef). Convención sobre los derechos del niño. 2006. Disponible en: <http://www.un.org/es/events/childrenday/pdf/derechos. pdf $>$. Acceso en: 20 nov. 2006.

García, J. El conocimiento y el currículum en la escuela: el reto de la complejidad. Buenos Aires: Homo Sapiens, 2007.

Gauthier, B. Recherche sociale. De la problématique à la collecte des données. SainteFoy: Presses de l'Université du Québec, 1998.

Gauthier, C.; Martineau, S.; Desbiens, J.-F. Pour un Théorie de la Pédagogie. Recherches Contemporaines sur le savoir des enseignants. Québec: Le Presses de l'Université Laval, 1997.

Guevara, T. Las últimas familias y costumbres mapuches. Santiago de Chile: Imprenta, Litografía i Encuadernación Barcelona, 1913.

Giroux, H. Los profesores como intelectuales. Barcelona: Paidós, 1990.

IzQuierdo, A. Espacio-temporalidad y omnijetividad. Una aproximación Epistemológica. Nómadas, Colombia, n. 11, p. 241-248, 1999.

Krippendorff, K. Metodología de análisis de contenido: teoría y práctica. Barcelona: Paidós, 1990.

Ley Indígena. Ley n. 19. 253. Temuco: CONADI, 1993.

Mato, D. No hay saber "universal”, la colaboración intercultural es imprescindible. Alteridades, Mexico, v. 18, n. 35, p. 101-116, 2008.

McMillan, J.; Schumacher, S. Investigación educativa. Madrid: Pearson Educación, 2005.

Ministerio de Educación (MINEDUC). Objetivos Fundamentales y Contenidos Minimos Obligatorios de la Educación Básica y Media: Actualización. Chile: Ministerio de Educación, 2009.

Morin, E. Articular los saberes ¿̇ué saberes enseñar en las escuelas? Buenos Aires: Ediciones Universidad del Salvador, 1998. 
Olıvé, L. Por una auténtica interculturalidad basada en el reconocimiento de la pluralidad epistemológica. In: CLACSO (Ed.). Pluralismo epistemológico. Bolivia: Muela del Diablo, 2009. p. 19-30.

Organização das Nações Unidas para a Educação, a Cî̂ncia e a Cultura (Unesco). Declaración de Naciones Unidas sobre los Derechos de los Pueblos Indígenas. Santiago: Oficina Regional de Educación para América Latina y el Caribe OREALC/UNESCO, 2007.

.La discriminación y el pluralismo cultural en la Escuela. Casos de Brasil, Chile, Colombia, México y Perú. Santiago: Oficina Regional de Educación para América Latina y el Caribe OREALC/UNESCO, 2005.

Perrenoud, P. Curriculum: le formel, le réel, le caché". In: Houssaye, J. La pédagogie: une encyclopédie pour aujourd'bui. Paris: ESF, 1994. p. 61-76.

Poblete, M. Comunidades mapuches de Panguipulli y educación: las primeras décadas del siglo XX. Revista Austral de Ciencias Sociales, n. 5, p. 15-27, 2001.

Quilaqueo, D.; Quintriqueo, S. Saberes educativos mapuches: un análisis desde la perspectiva de los kimches. Polis, Bolívia, v. 9, n. 26, p. 337-360, 2010.

Quilaqueo,D.; Quintriqueo, S.; CÁrdenas, P.Educación, currículum e interculturalidad: elementos sobre formación de profesores en contexto mapuche. Santiago: Frasis, 2005.

Quintriqueo, S. Epistemología del conocimiento mapuche y el escolar: un análisis desde la pedagogía intercultural, la geografía y sociología educacional. Proyecto FONDECYT 1110489. Chile, 2011-2013.

. Implicancias de un modelo curricular monocultural en contexto mapuche. Santiago: Gráfica LOM, 2010.

. Conocimientos geográficos y territoriales mapuches: una base para la formulación de contenidos educativos interculturales pertinentes y contextualizados. Proyecto FONDECYT 1140490. Chile, 2014-2017.

Quintriqueo, S.; CÁrdenAs, P. Educación intercultural en contextos mapuches: hacia una articulación entre el conocimiento mapuche y conocimiento disciplinario en ciencia. In: Quilaqueo, D.; Fernández, C.; Quintriqueo, S. Interculturalidad en contexto mapuche. Neuquén: Educo, 2010. p. 89-126.

Quintriqueo, S. et al. Conocimientos culturales como contenidos de la educación familiar mapuche. Alpha, n. 40, p. 131-146, 2015.

Quintriqueo, S. et al. Formación del profesorado en educación intercultural en América Latina. El caso de Chile. Revista Electrónica Interuniversitaria de Formación del Profesorado, v. 17, n. 2, p. 201-217, 2014.

Quintriqueo, S.; Maheux, G. Exploración del conocimiento sobre la relación de parentesco como contenido educativo para un currículum escolar intercultural en comunidades mapuche. Revista de Psicología, Santiago, v. 13, n. 1, p. 73-91, 2004.

Quintriqueo, S.; Muñoz, G. Conocimiento mapuche y su relación con el conocimiento escolar. In: Congreso Nacional de Ciencias Sociales y Educación, 1., 2012, Chile. Actas... 2012.

Quintriqueo, S.; Quilaqueo, D.; Riquelme, E. Epistemological distance of schooling in the Mapuche context in the Araucania Region of Chile. 2014. (no prelo). 
Quintriqueo, S.; Torres, H. Distancia entre el conocimiento mapuche y el conocimiento escolar en contexto mapuche. Revista Electrónica de Investigación Educativa, v. 14, n. 1, p. 16-33, 2012.

Rockwell, E. La escuela cotidiana. México: Fondo de Cultura Económica, 1995.

Rodríguez, G.; Gil, J.; García, E. Metodología de la investigación cualitativa. Málaga: Aljibe, 1999.

Ruiz, C. El Enfoque Multimétodo en la Investigación Social y Educativa: Una Mirada desde el Paradigma de la Complejidad. Revista de Filosofía y Sociopolítica de la Educación, Venezuela, v. 4, n. 8, p. 13-28, 2008.

Schmelkes, S. Las universidades interculturales en México: ¿una contribución a la equidad en educación superior? In: Conference on Ethnicity, Race, And Indigenous Peoples in Latin America and The Caribbean, 1., 2008, San Diego. Mayo 2008.

\section{SOBRE LOS AUTORES}

Segundo Enrique Quintriqueo Millan es doctor en educación por la Universidad de Extremadura (España). Profesor de la Universidad Católica de Temuco (Chile).

E-mail: squintri@uct.cl

Enrique Hernán Riquelme Mella es doctor en educación por la Universidad Autónoma de Madrid (España). Profesor de la Universidad Católica de Temuco (Chile).

E-mail: eriquelme@uct.cl

Daniel Quilaqueo-Rapimán es doctor en Sociología por la Université de la Sorbonne (Francia). Profesor de la Universidad Católica de Temuco (Chile). E-mail:dquilaq@uct.cl

Soledad Morales Saavedra es magister en Psicología por la Universidad de la Frontera de Temuco (Chile). Profesora de la Universidad Católica de Temuco (Chile).

E-mail:smorales@uct.cl

Maritza Cecilia Gutierrez Surjan es doctora en ciencias por la Universidad Austral de Chile (UACH). Profesora de la Universidad Católica de Temuco (Chile).

E-mail:mcgutierrez@uct.cl 